\title{
The Effectiveness of Preoperative Trimetazidine on Myocardial Preservation in Coronary Artery Bypass Graft Patients: A Systematic Review and Meta-Analysis
}

\author{
Nan Zhang ${ }^{a}$ Jiayan Lei ${ }^{c}$ Qing Liu ${ }^{b}$ Wei Huang ${ }^{a}$ Hua Xiao $^{a}$ Han Lei ${ }^{a}$ \\ ${ }^{a}$ Department of Cardiology and ${ }^{b}$ Center for Clinical Research, The First Affiliated Hospital of Chongqing Medical \\ University, and 'The First Affiliated Hospital of Chongqing Medical University, Chongqing, China
}

\author{
Key Words \\ Coronary artery bypass graft $\cdot$ Trimetazidine $\cdot$ Myocardial \\ preservation - Myocardium
}

\begin{abstract}
Background: Coronary artery bypass grafting (CABG) is a key and effective surgical treatment modality for coronary artery disease. Unfortunately, ischemia-reperfusion injury during and after CABG can lead to reversible and irreversible myocardial damage. Trimetazidine [1-(2,3,4-trimethoxybenzyl) piperazine dihydrochloride] is a metabolic anti-ischemic agent with demonstrated cardioprotective effects; however, its effects with respect to myocardial preservation in CABG patients remain unclear. Methods: We conducted a systematic review and meta-analysis of randomized controlled trials (RCTs) to investigate the effectiveness of myocardial preservation of preoperative trimetazidine therapy in CABG patients by assessing the postoperative levels of several blood-based biochemical markers of myocardial injury, including creatine kinase (CK), creatine kinase-muscle and brain (CK-MB), creatine phosphokinase (CPK), troponin $\mathrm{T}$ (TnT) and troponin I (Tnl). The RCTs were classified into two subgroup analyses by the timing of sample collection (either
\end{abstract}

$\leq 12$ or $>12 \mathrm{~h}$ after CABG). Results: Six RCTs were finally included in the meta-analysis. The pooled effect sizes showed significantly lower postoperative levels of CK, CK-MB, TnT and $\mathrm{Tnl}$ in the trimetazidine-treated CABG patients relative to control CABG patients. However, there were no significant differences in the postoperative CPK levels between trimetazidine-treated $C A B G$ patients relative to control $C A B G$ patients. In both the $\leq 12$ and $>12 \mathrm{~h}$ post-CABG subgroup analyses, significant differences in CK, CK-MB, TnT and Tnl were detected between the trimetazidine-treated $C A B G$ patients relative to control CABG patients. Conclusions: Preoperative trimetazidine therapy appears to have a positive effect on myocardial preservation in CABG patients.

(c) 2015 S. Karger AG, Basel

\section{Introduction}

Coronary artery bypass grafting (CABG) is a key and effective surgical treatment modality for coronary artery disease [1]. Unfortunately, ischemia-reperfusion injury during and after CABG can lead to reversible and irreversible myocardial damage [2]. Clinically, ischemia-reperfusion injury after CABG can manifest as myocardial

\section{KARGER 125}

(c) 2015 S. Karger AG, Base

$0008-6312 / 15 / 1312-0086 \$ 39.50 / 0$

E-Mail karger@karger.com

www.karger.com/crd
Dr. Han Lei

Department of Cardiology

The First Affiliated Hospital of Chongqing Medical University

1 Youyi Road, Yuzhong, Chongqing 400016 (China)

E-Mail zn127127@126.com 
stunning, perioperative myocardial infarction, low cardiac output or arrhythmia. In patients dying soon after CABG, histopathological evidence of ischemia-reperfusion is detected in $25-45 \%$ postmortem [3, 4]. Furthermore, biochemical evidence of myocardial injury (e.g. elevated levels of circulating creatine kinase-muscle and brain, CK-MB, and/or troponin) has been clearly linked with adverse events after CABG [5]. Therefore, myocardial preservation is a key issue for patients undergoing CABG.

Trimetazidine [1-(2,3,4-trimethoxybenzyl)piperazine dihydrochloride] is a metabolic anti-ischemic agent with demonstrated cardioprotective effects that does not alter cardiac hemodynamics [6]. Accordingly, meta-analyses of randomized controlled trials (RCTs) have validated the beneficial cardioprotective effects of trimetazidine therapy in patients with stable angina pectoris and congestive heart failure (CHF) [7-11]. Although trimetazidine shows promise as an anti-ischemic agent, its effects with respect to myocardial preservation in CABG patients remain unclear.

Therefore, here we conducted a systematic review and meta-analysis of RCTs to investigate the effectiveness of myocardial preservation of pre-operative trimetazidine therapy in CABG patients by assessing the postoperative levels of several blood-based biochemical markers of myocardial injury, including creatine kinase (CK), CK$\mathrm{MB}$, creatine phosphokinase $(\mathrm{CPK})$, troponin $\mathrm{T}(\mathrm{TnT})$ and troponin I (TnI) [12].

\section{Methods}

This study was performed in accordance with the PRISMA recommendations for systematic reviews and meta-analyses [13] and the Cochrane handbook for systematic reviews of interventions [14]. The protocol of this study has not been published.

\section{Search Strategy}

We performed a systematic search for RCTs investigating the use of preoperative trimetazidine in CABG patients through the following databases: MEDLINE, PubMed, Web of Science and China National Knowledge Infrastructure with the following key words and MeSH headings: ('coronary artery bypass graft' OR 'CABG' OR 'coronary bypass surgery') AND ('trimetazidine' OR 'vastarel' OR 'vasorel') AND ('randomized controlled trial' OR 'RCT'). The time period was restricted from January 1, 1971, to December 31, 2013, and the languages were restricted to English and Chinese. In addition, all references of the related reviews and articles were searched to find additional studies.

\section{Study Selection}

References were evaluated by two individual investigators (N.Z., W.H.) using the following predefined inclusion and exclusion criteria.

Preoperative Trimetazidine on Myocardial Preservation in CABG Patients
Inclusion Criteria

RCTs conducted in CABG patients treated with trimetazidine (i.e. RCTs directly comparing trimetazidine and placebo in CABG patients) regardless of sample size.

\section{Exclusion Criteria}

(i) Failure to report a relevant data outcome (i.e. postoperative circulating levels of CK, CK-MB, CPK, TnT or TnI) and (ii) reviews, editorials, comments or reports from scientific sessions or discussions.

Decision for inclusion was made on consensus. Any conflict between the reviewers was resolved by an author group discussion, after which the primary authors made the final decision. Evaluation was based on title and abstract whenever available. Full-text articles from potentially relevant references were obtained in electronic or printed format and re-evaluated for inclusion by the same investigators as before. The PICOS (patients, interventions, comparators, outcomes and settings) method was used to assess whether the references fully complied with the inclusion and exclusion criteria [15]. As a full-text article was required for this systematic review and meta-analysis, references whose full texts we could not acquire either electronically or as printed copies from our medical library were excluded. Multiple reports from a single study were considered as one study.

\section{Bias Evaluation}

As instructed in the Cochrane handbook for systematic reviews of interventions [14], the investigators performed an evaluation of bias rather than of methodological quality. Studies included were evaluated for bias using methods described in the Cochrane handbook. The following 6 dimensions were considered in the bias assessment tool: allocation sequence generation, allocation concealment, blinding of participants, incomplete outcome data, selective outcome reporting, and other sources of bias. Evaluation was done by two independent assessors (N.Z., W.H.) to improve the validity. In addition, publication bias was assessed by visual inspection of funnel plots.

\section{Data Extraction}

Data on key characteristics of the RCTs were extracted by two independent researchers (N.Z., W.H.), including country, sample size, age, preoperative trimetazidine course of therapy (durations and routes), measured outcomes (postoperative circulating levels of CK, CK-MB, CPK, TnT and TnI) and the postoperative follow-up period (mean hours), using an Excel data extraction form. Any conflict between the reviewers was solved by a group discussion, after which the primary authors made the final decision.

\section{Meta-Analyses}

Data were entered into Review Manager 5.0 software to conduct the heterogeneity analysis and meta-analysis (Review Manager, version 5 for Windows, 2008). We analyzed myocardial preservation in $\mathrm{CABG}$ patients based on their postoperative levels of CK, CK-MB, CPK, TnT and TnI. Since continuous data from different scales were extracted, the standardized mean difference (SMD) was calculated for effect size based on sample size and $95 \%$ confidence intervals (CIs) for each study, and for the pooled studies using variance analysis [16]. A 2-sided $p$ value of less than 0.05 was deemed significant for all analyses. 
Fig. 1. Flowchart of study selection based on initial literature search findings followed by application of the inclusion and exclusion criteria.

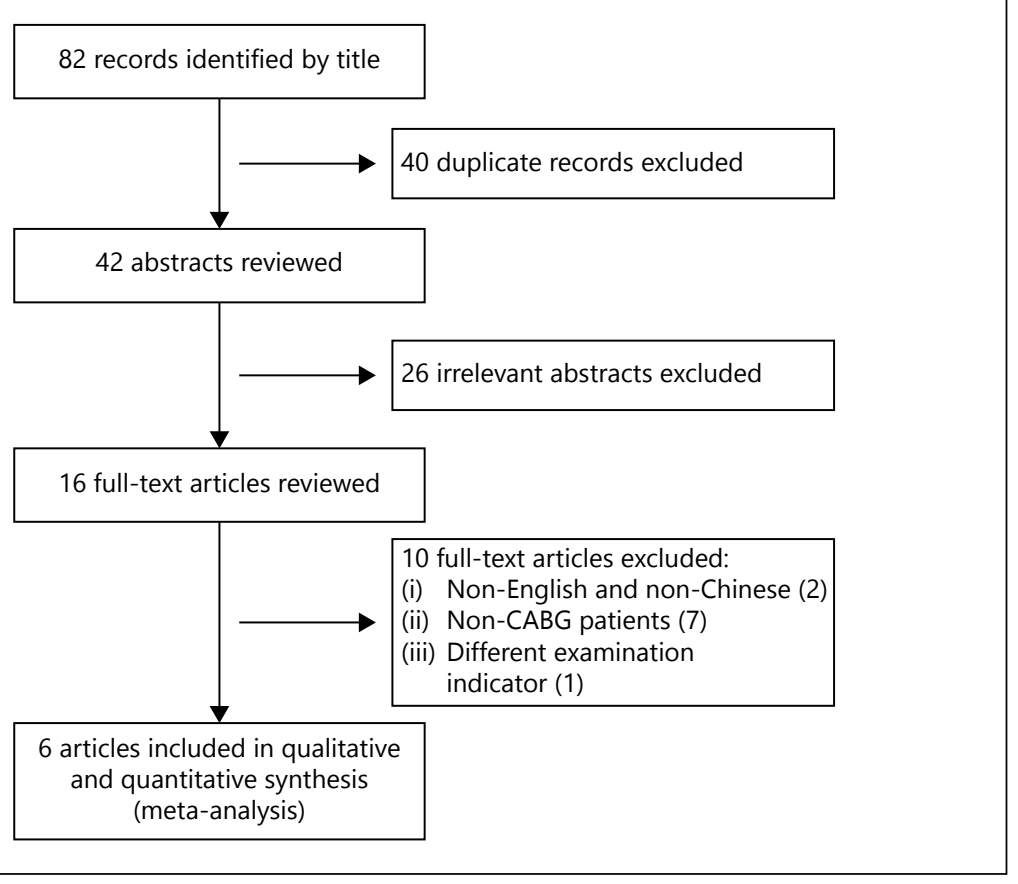

The fixed effect model was used to pool data if there was no heterogeneity detected; otherwise, the random effects model was used. $\mathrm{I}^{2}$ was the percentage of variation attributed to heterogeneity with an $\mathrm{I}^{2}$ statistic of $25-50 \%$ considered low, $50-75 \%$ considered moderate and $\geq 75 \%$ considered high. Heterogeneity was considered significant for $\mathrm{p}$ values of Cochran's Q statistic of less than 0.10 and $\mathrm{I}^{2}$ values of greater $50 \%[17,18]$. If heterogeneity was detected, we conducted a sensitivity analysis to verify the reliability of the meta-analysis results by excluding certain studies and then recalculating the pooled estimates for the remaining studies.

\section{Results}

The flowchart of study selection is provided in figure 1 . The initial literature search yielded 82 records, of which 6 RCTs remained after removal of duplicate records and application of all inclusion and exclusion criteria [19-24]. In the following subgroup meta-analysis based on each of the postoperative myocardial injury markers (i.e. CK, CK-MB, CPK, TnT and TnI), these 6 RCTs were classified into 2 subgroups by the timing of sample collection (either $\leq 12$ or $>12 \mathrm{~h}$ after $\mathrm{CABG}$ ).

\section{Meta-Analysis: Postoperative CK}

Figure 2 shows a Forest plot evaluating preoperative trimetazidine therapy by postoperative circulating $\mathrm{CK}$ levels. With respect to postoperative circulating CK lev- els, Iskesen et al. [22] and Vedrinne et al. [21] each reported 3 data outcomes. There was high heterogeneity $\left(\mathrm{I}^{2}=99 \%, \mathrm{p}<0.00001\right)$; thus, the random effects model was used.

The pooled effect size showed significant differences in postoperative $\mathrm{CK}$ levels $(\mathrm{SMD}=-138.99,95 \% \mathrm{CI}=$ -219.83 to $-58.16, \mathrm{p}=0.0008$ ) between the trimetazidine and control groups. In the $\leq 12 \mathrm{~h}$ postoperation subgroup analysis, all RCTs with the exception of Iskesen et al. [22], subgroups $\mathrm{A}$ and $\mathrm{B}$, showed significant differences in postoperative CK levels between the trimetazidine and control groups. In the $>12 \mathrm{~h}$ postoperation subgroup analysis, the 2 RCTs showed significant differences in postoperative CK levels between the trimetazidine and control groups.

\section{Meta-Analysis: Postoperative CK-MB}

Figure 3 shows a Forest plot evaluating pre-operative trimetazidine therapy by post-operative circulating CK-MB levels. With respect to postoperative circulating CK-MB levels, Iskesen et al. [22] reported 2 data outcomes, Sher-i-Murtaza et al. [19] reported 3 data outcomes, Tünerir et al. [20] reported 4 data outcomes, Vedrinne [21] reported 5 data outcomes, and Wang et al. [24] reported 2 data outcomes. There was high heterogeneity $\left(\mathrm{I}^{2}=95 \%, \mathrm{p}<0.00001\right)$; thus, the random effects model was used. 


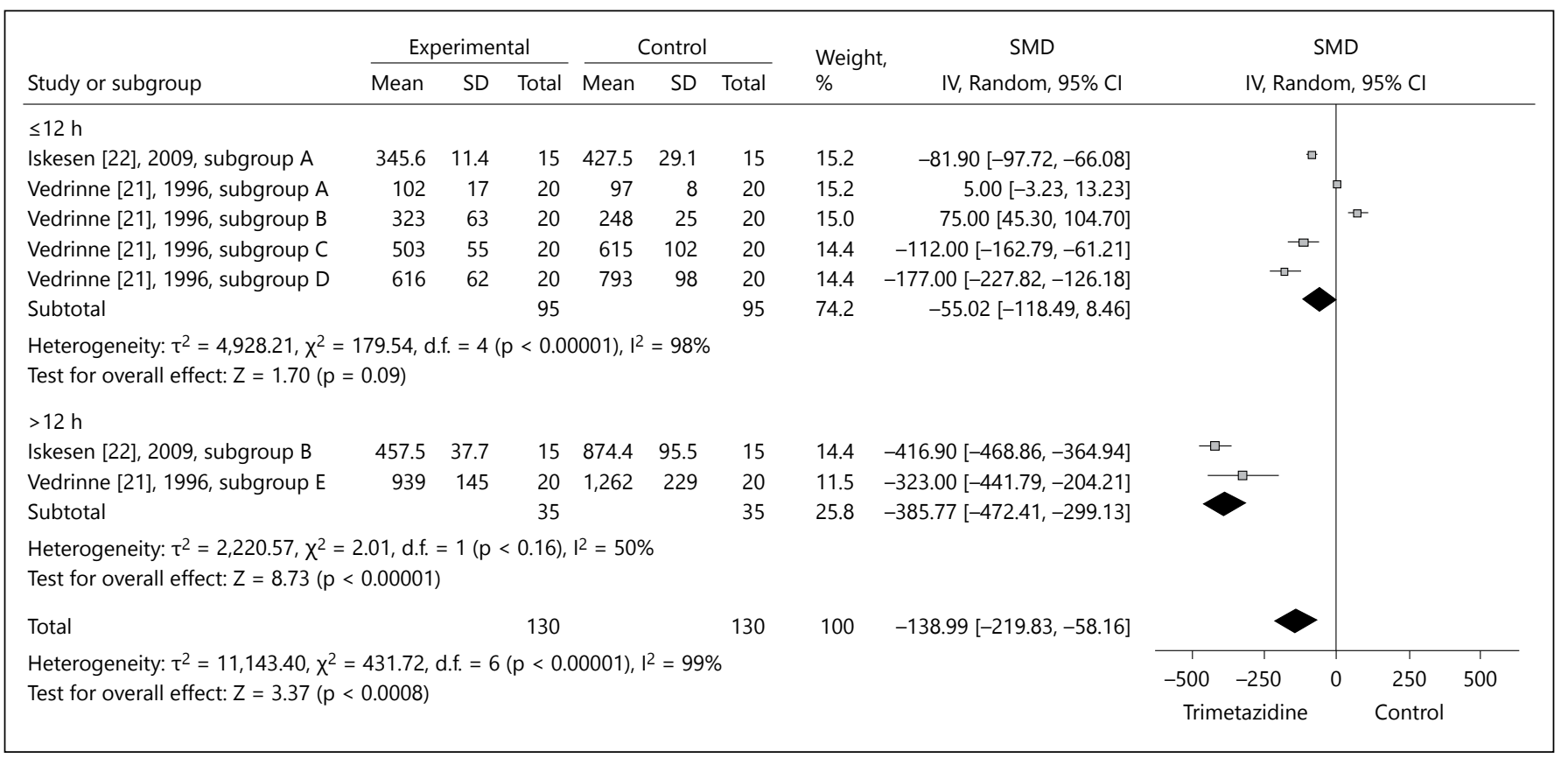

Fig. 2. Forest plot of RCTs evaluating preoperative trimetazidine and postoperative circulating CK levels. The squares and horizontal lines correspond to the SMDs and 95\% CIs, respectively.

The pooled effect size showed significant differences in postoperative CK-MB levels $(\mathrm{SMD}=-2.30,95 \% \mathrm{CI}=$ -3.01 to $-1.58, \mathrm{p}<0.0001)$ between the trimetazidine and control groups. In the $\leq 12 \mathrm{~h}$ postoperation subgroup analysis, all RCTs with the exception of that of Sher-iMurtaza et al. [19], subgroups A and B, showed significant differences in postoperative CK-MB levels between the trimetazidine and control groups. In $>12 \mathrm{~h}$ postoperation subgroup analysis, all RCTs with the exception of that of Sher-i-Murtaza et al. [19], subgroup C, showed significant differences in postoperative CK-MB levels between the trimetazidine and control groups.

\section{Meta-Analysis: Postoperative CPK}

Figure 4 shows a Forest plot evaluating preoperative trimetazidine therapy by postoperative circulating CPK levels. With respect to postoperative circulating CPK levels, Sher-i-Murtaza et al. [19] reported 3 data outcomes. There was no heterogeneity detected $\left(\mathrm{I}^{2}=0 \%, \mathrm{p}=0.67\right)$; thus, the fixed effects model was used.

The pooled effect size showed no significant differences in postoperative CPK levels $(\mathrm{SMD}=16.63,95 \% \mathrm{CI}=$ -63.49 to $-96.74, \mathrm{p}=0.068$ ) between the trimetazidine and control groups. In both the $\leq 12$ and $>12$ h postoperation subgroup analyses, Sher-i-Murtaza et al. [19],
The area of the squares reflects the study specific weight (inverse of the variance). The diamonds represent the pooled SMDs and 95\% CIs.

subgroups A-C, showed no significant differences in postoperative $\mathrm{CPK}$ levels between the trimetazidine and control groups.

\section{Meta-Analysis: Postoperative TnT}

Figure 5 shows a Forest plot evaluating preoperative trimetazidine therapy by postoperative circulating TnT levels. With respect to postoperative circulating TnT levels, Iskesen et al. [22] reported 3 data outcomes, Sher-iMurtaza et al. [19] reported 3 data outcomes, and Tünerir et al. [20] reported 4 data outcomes. There was high heterogeneity ( $\left.\mathrm{I}^{2}=99 \%, \mathrm{p}<0.00001\right)$; thus, the random effects model was used.

The pooled effect size showed a significant difference in postoperative $\mathrm{TnT}$ levels $(\mathrm{SMD}=-1.42,95 \% \mathrm{CI}=$ -1.99 to $-0.84, \mathrm{p}<0.00001$ ) between the trimetazidine and control groups. In both the $\leq 12$ and $>12 \mathrm{~h}$ postoperation subgroup analyses, Iskesen et al. [22] and Tünerir et al. [20] showed significant differences between the trimetazidine and control groups.

\section{Meta-Analysis: Postoperative TnI}

Figure 6 shows a Forest plot evaluating preoperative trimetazidine therapy by postoperative circulating $\mathrm{TnI}$ levels. With respect to postoperative circulating TnI lev- 


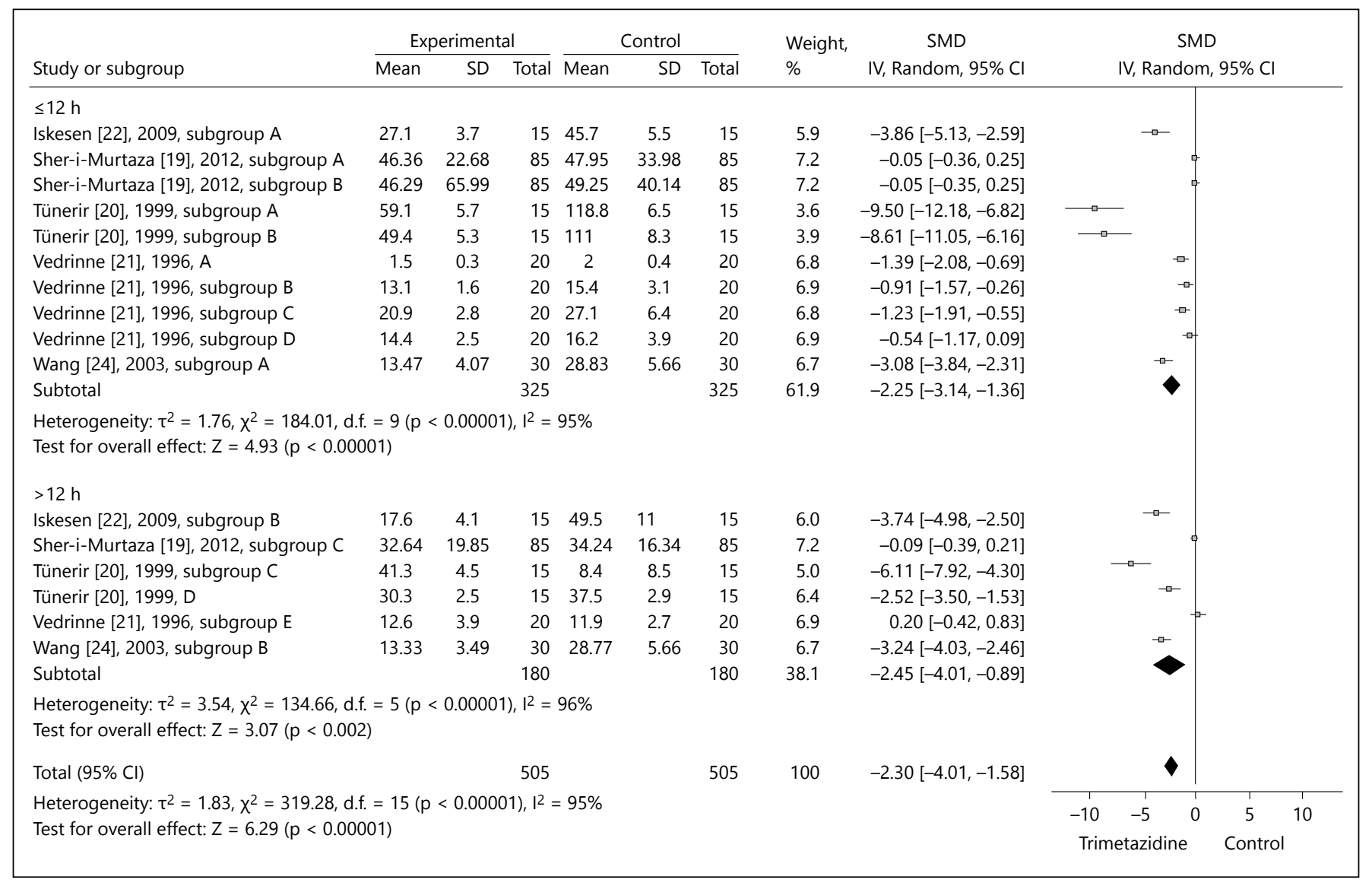

Fig. 3. Forest plot of RCTs evaluating preoperative trimetazidine and postoperative circulating CK-MB levels. The squares and horizontal lines correspond to the SMDs and 95\% CIs, respectively.
The area of the squares reflects the study specific weight (inverse of the variance). The diamonds represent the pooled SMDs and $95 \%$ CIs.

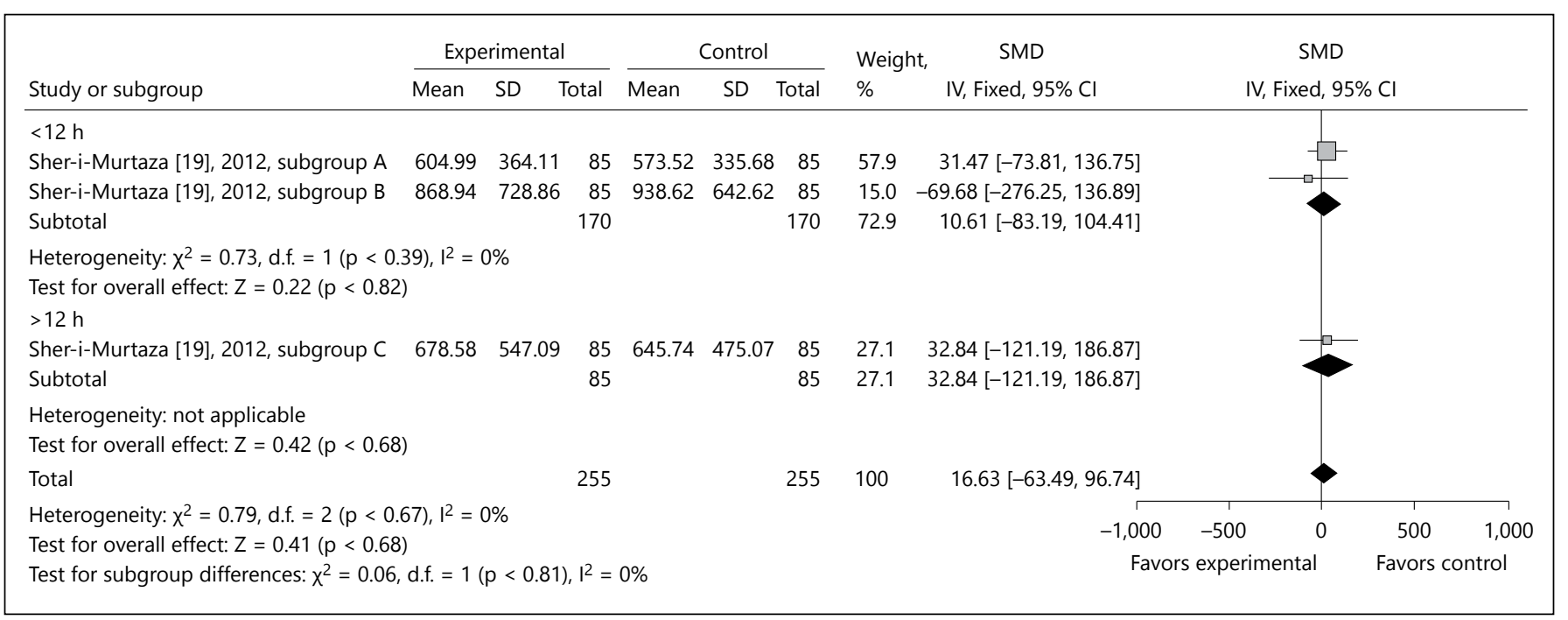

Fig. 4. Forest plot of RCTs evaluating preoperative trimetazidine and postoperative circulating CPK levels. The squares and horizontal lines correspond to the SMDs and 95\% CIs, respectively.
The area of the squares reflects the study specific weight (inverse of the variance). The diamonds represent the pooled SMDs and 95\% CIs. 


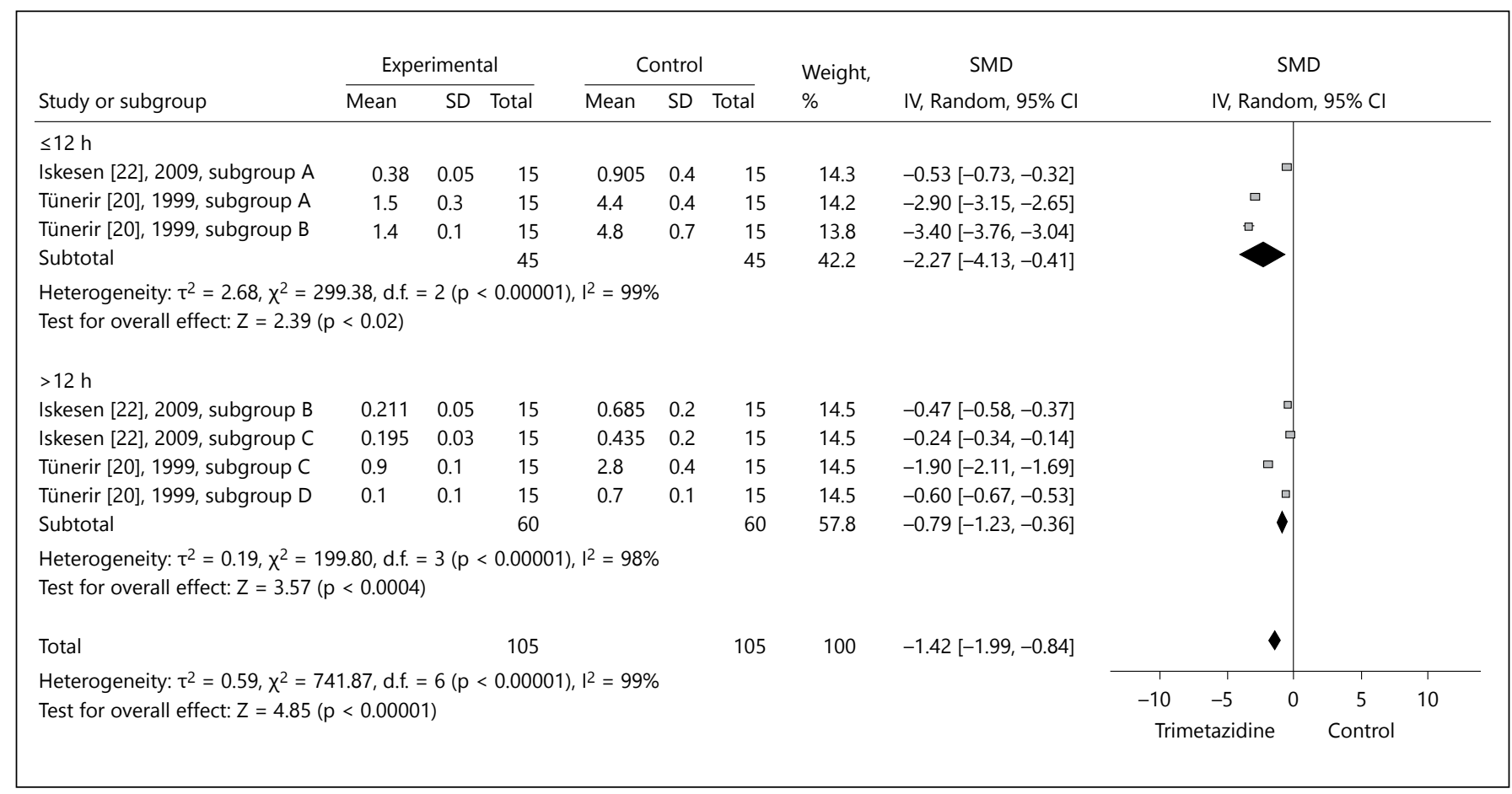

Fig. 5. Forest plot of RCTs evaluating preoperative trimetazidine and postoperative circulating TnT levels. The squares and horizontal lines correspond to the SMDs and 95\% CIs, respectively.
The area of the squares reflects the study specific weight (inverse of the variance). The diamonds represent the pooled SMDs and $95 \%$ CIs.

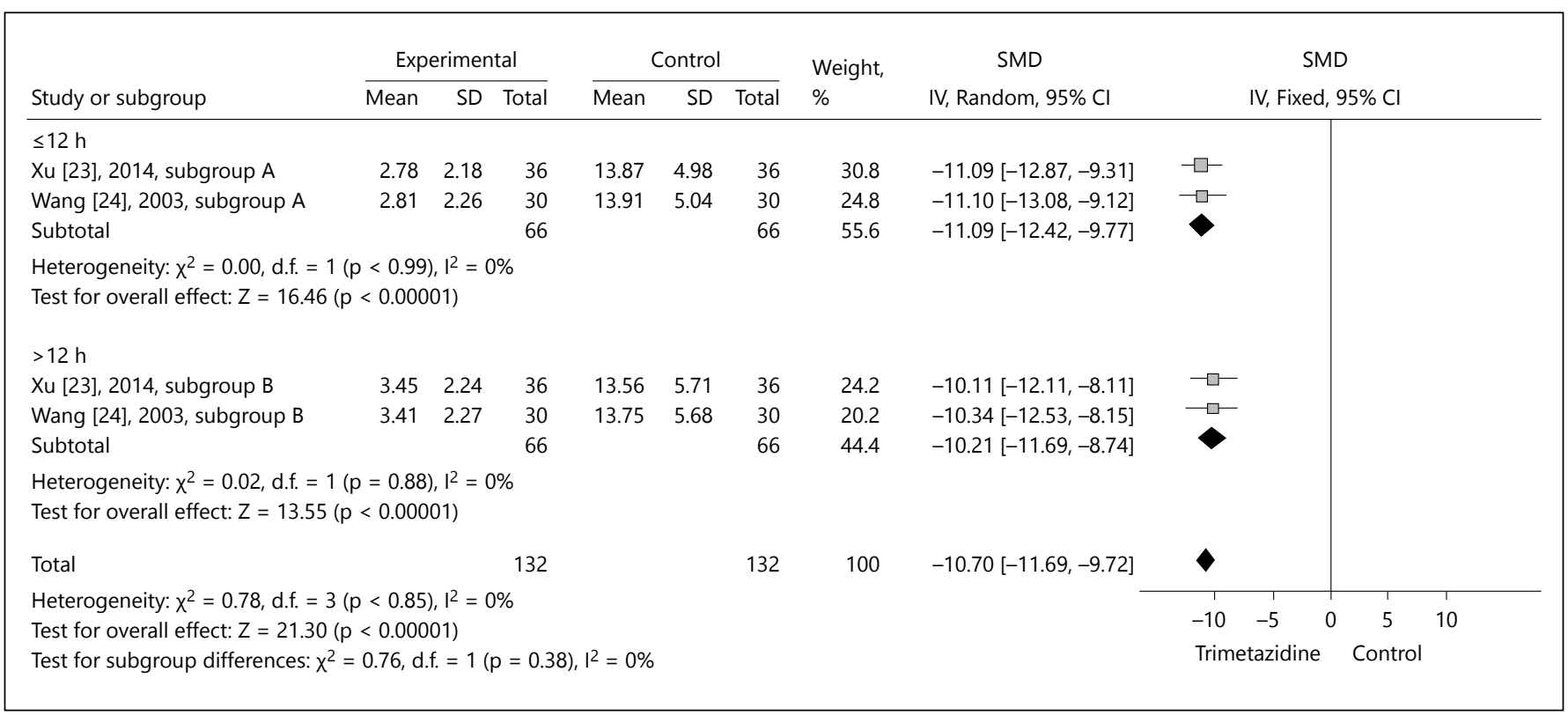

Fig. 6. Forest plot of RCTs evaluating preoperative trimetazidine and postoperative circulating TnI levels. The squares and horizontal lines correspond to the SMDs and 95\% CIs, respectively.
The area of the squares reflects the study specific weight (inverse of the variance). The diamonds represent the pooled SMDs and 95\% CIs. 


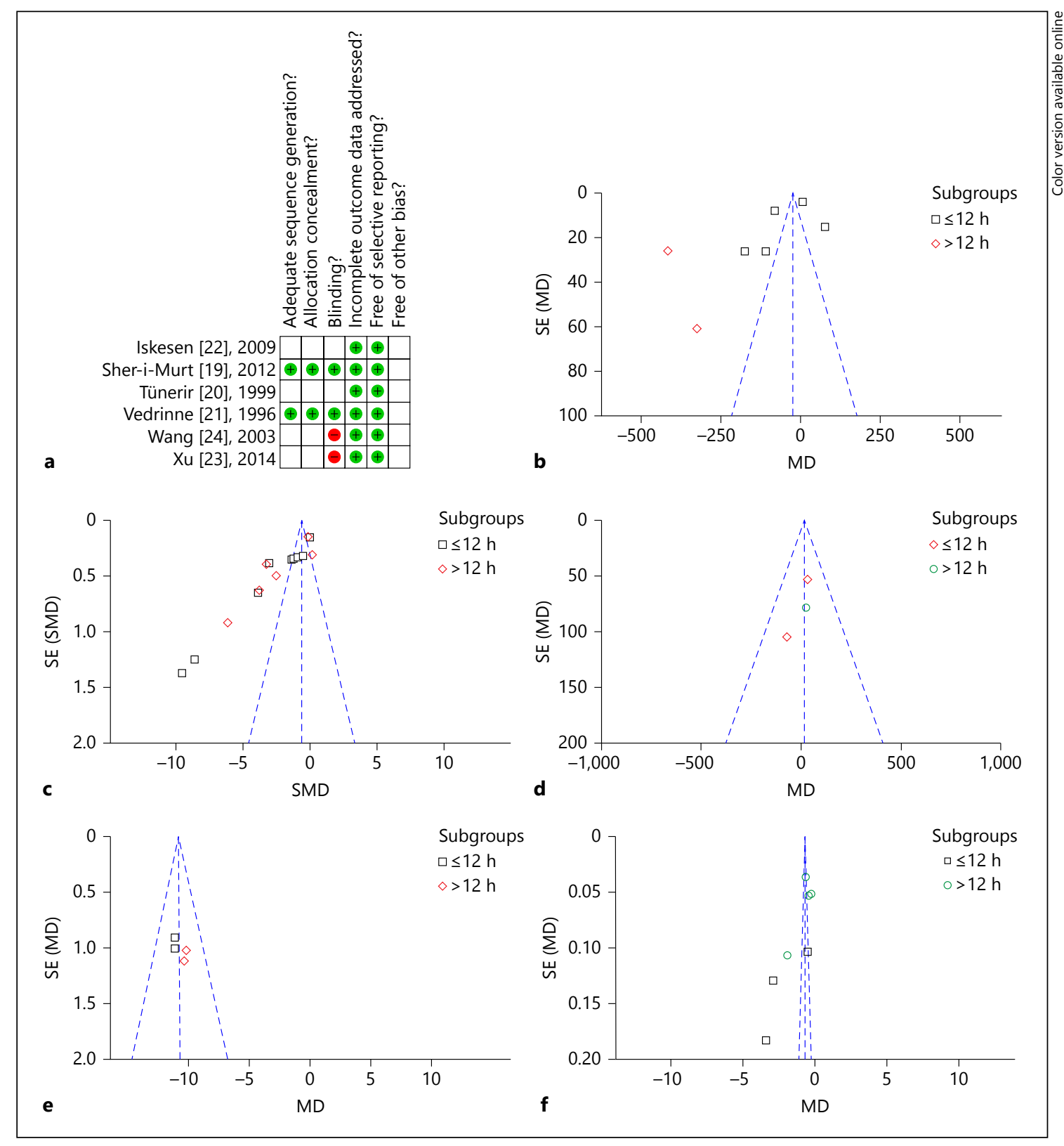

Fig. 7. Bias evaluation. a Results of the Cochrane bias assessment tool. b Funnel plot for CK. c Funnel plot for CK-MB. d Funnel plot for CPK. e Funnel plot for TnI. f Funnel plot for TnT. Funnel plots indicate the particular study's timing of sample collection (i.e. $\leq 12$ or $>12 \mathrm{~h}$ ).

els, $\mathrm{Xu}$ et al. [23] reported 2 data outcomes, and Wang et al. [24] reported 2 data outcomes. There was no heterogeneity detected $\left(\mathrm{I}^{2}=0 \%, \mathrm{p}=0.85\right)$; thus, the fixed effects model was used.

The pooled effect size showed a significant difference in postoperative $\mathrm{TnT}$ levels $(\mathrm{SMD}=-10.7,95 \% \mathrm{CI}=$ -11.69 to $-9.72, \mathrm{p}<0.00001)$ between the trimetazidine and control groups. In both the $\leq 12$ and $>12 \mathrm{~h}$ postop- eration subgroup analyses, $\mathrm{Xu}$ et al. [23] and Wang et al. [24] showed significant differences between the trimetazidine and control groups.

\section{Bias Evaluation}

With respect to bias, the $2 \mathrm{TnI}$ studies $[23,24]$ failed to blind participants, which may have been a source of bias in the TnI meta-analysis (fig. 7a). Accordingly, visual inspec- 
tion of the funnel plots revealed slight asymmetry in these TnI studies as well (fig. 7f), suggesting possible publication bias in the TnI meta-analysis. The remaining funnel plots did not appear to be grossly asymmetrical (fig. 7a-e).

\section{Discussion}

Here, we conducted a systematic review and metaanalysis of placebo-controlled RCTs to investigate the effectiveness of myocardial preservation of preoperative trimetazidine therapy in $\mathrm{CABG}$ patients by assessing the postoperative levels of several blood-based biochemical markers of myocardial injury. The pooled effect sizes showed significantly lower postoperative levels of CK, CK-MB, TnT and TnI in trimetazidine-treated CABG patients relative to control CABG patients. However, there were no significant differences in the postoperative $\mathrm{CPK}$ levels between the trimetazidine-treated CABG patients relative to control CABG patients. In sum, preoperative trimetazidine therapy appears to have a positive effect on myocardial preservation in CABG patients.

\section{Trimetazidine's Cardioprotective Effects}

Trimetazidine is an anti-ischemic agent with demonstrated cardioprotective effects that works through several mechanisms. First, it inhibits long-chain mitochondrial 3-ketoacyl coenzyme A thiolase, which plays a key role in $\beta$-oxidation in cardiomyocytes [25]. This action switches cardiomyocyte metabolism from free fatty acid oxidation to glucose oxidation, which is more efficient at oxygen consumption and ATP production [25]. Second, through improving energy insufficiency, trimetazidine reduces sodium accumulation in cardiomyocyte cytoplasm, decreases reactive oxygen species formation and reduces neutrophil infiltration [25]. Third, trimetazidine reduces collagen accumulation, cardiac fibroblasts' connective tissue growth factor expression, nicotinamide adenine dinucleotide phosphate oxidase levels and reactive oxygen species production [25]. Fourth, during reperfusion following an acute ischemic episode, trimetazidine also improves the sarcolemma's mechanical resistance to edema-induced mechanical stress [25].

Clinically, trimetazidine has been shown to be effective in angina pectoris patients across several meta-analyses. The meta-analysis of Marzilli and Klein [26] of 12 trials consisting of 868 patients found that trimetazidine increased exercise duration to 1-mm ST segment depression on the exercise test and reduced anginal episodes. That of Ciapponi et al. [27] of 23 trials with 1,378 patients

Preoperative Trimetazidine on Myocardial Preservation in CABG Patients showed that trimetazidine reduced anginal attacks, nitroglycerine tablet consumption and improved exercise time to $1-\mathrm{mm}$ segment depression on the exercise test. The network meta-analysis of Danchin et al. [7] of 218 trials with 19,028 patients showed trimetazidine improved exercise tolerance and weekly angina episodes; moreover, trimetazidine displayed similar anti-ischemic effects to dihydropyridines, long-acting nitrates, nicorandil and ranolazine. Most recently, the meta-analysis of Belsey et al. [28] also showed that trimetazidine used in conjunction with first-line $\beta$-blockers or calcium channel blockers showed improvement across several clinical outcomes.

In addition, trimetazidine has been shown to be effective in CHF patients across 3 meta-analyses. The metaanalysis of Gao et al. [29] of 17 RCTs with $955 \mathrm{CHF}$ patients showed trimetazidine use was associated with increased exercise tolerance, New York Heart Association (NYHA) functional class reduction, improved left ventricular ejection fraction (LVEF), a reduced rate of cardiovascular events and hospitalizations, and reduction in overall mortality. The meta-analysis of Zhang et al. [10] of 16 RCTs with 884 patients demonstrated that trimetazidine use was associated with improved LVEF, increased exercise tolerance, reduced NYHA functional class, decreased left ventricular end-systolic volume and left ventricular end-diastolic volume, lowered B-type natriuretic peptide levels and a reduced rate of cardiovascular hospitalization; however, no reduction in overall mortality was observed [10]. Most recently, the meta-analysis of Zhou and Chen [9] of 19 RCTs with 994 CHF patients showed trimetazidine improved left ventricular ejection fraction and NYHA functional class while decreasing left ventricular end-systolic volume, left ventricular end-diastolic volume, hospitalization for cardiac causes, B-type natriuretic peptide and Creactive protein; however, trimetazidine did not significantly affect exercise duration or all-cause mortality.

\section{Measuring Myocardial Preservation with Cardiac Markers}

Measuring myocardial preservation at the cellular level is a challenge. Here, in order to assess trimetazidine's effectiveness at myocardial preservation in CABG patients, we analyzed the levels of 5 commonly used biochemical markers of myocardial injury (CK, CK-MB, $\mathrm{Tn} \mathrm{T}$ and $\mathrm{TnI}$ ) as a proxy for myocardial preservation. In order to serve as a proxy for myocardial preservation, the ideal characteristics of a cardiac marker should be as follows: (i) the cardiac marker should be released into the circulation; (ii) the release of the cardiac marker into the circulation should occur in concert with and in propor- 
tion to the extent of myocardial cell death; (iii) the cardiac marker should possess a high myocardial concentration combined with a relatively low nonmyocardial concentration, which ensures a high myocardial specificity, and (iv) the cardiac marker should not associate or bind with other circulating factors in the bloodstream [30,31]. As compared to CK-MB that is not unique to the heart and is found in skeletal muscle and the gastrointestinal tract and can form complexes with circulating immunoglobulins, TnI and TnT are unique to the cardiac myofibril's contractile apparatus, have a higher percentage released into the bloodstream after cardiac injury, do not form immunoglobulin complexes, and display a superior persistence in the bloodstream owing to the slow release and slow degradation with a half-life of about $2 \mathrm{~h}$, allowing for improved clinical detection after a cardiac event [31]. On this basis, the troponins (TnT, TnI) have been shown to be better predictors of post-CABG mortality than CK-MB; clinical studies comparing CK-MB with TnI and TnT have demonstrated that troponins are superior predictors of post-CABG mortality [32-34].

Here, we showed that both troponins (TnT, TnI) as well as CK-MB were all significantly reduced after trimetazidine therapy, which logically suggests that trimetazidine therapy improves post-CABG mortality. However, longer-term clinical trials on post-CABG patients that monitor all key cardiac biomarkers in addition to cardiovascular and all-cause mortality are required to validate this hypothesis.

\section{Timing of Sample Collection}

The timing of sample collection is also a key issue. After an acute myocardial injury, CK-MB and TnI take $2-8 \mathrm{~h}$ to increase above the upper reference limit, peaking between 14 and $36 \mathrm{~h}$, and then remaining elevated 3-7 days after injury; TnT mimics the early release kinetics of TnI but can remain elevated for as long as 3 weeks [35]. However, the post-CABG release kinetics of these cardiac markers is complicated by other factors including preoperative myocardial injury and reperfusion injury, mediastinal blood retransfusion, graft failure, skeletal muscle injury (which disproportionally affects CK-MB levels), continuous extended release from degrading cardiac myofibrils (which disproportionally affects troponin levels), renal failure (which disproportionally affects troponin levels) and ischemic modification of molecules (which disproportionally affects TnI levels) [36]. Despite this physiological complexity, early cardiac marker release has been shown to predict length of stay in the hospital and adverse outcomes, but the majority of studies show that later release of cardiac markers $(>12 \mathrm{~h}$ after cardiac event) are more clinically relevant [36].

Here, we divided the meta-analysis into early sample collection ( $\leq 12 \mathrm{~h}$ after operation) and late sample collection ( $>12 \mathrm{~h}$ after operation) in order to better assess the aforementioned effects of differential release kinetics. Comparing the pooled SMDs from the two timings on a marker-by-marker basis, we observed that CK (early-55.02 vs. late -385.77 ) and CK-MB (early -2.25 vs. late -2.45 ) showed more prominent SMDs in trimetazidine-treated CABG patients after $12 \mathrm{~h}$ while TnT (early -2.27 vs. late -0.79 ) and TnI (early -11.09 vs. late -10.21 ) showed more prominent SMDs in trimetazidine-treated CABG patients before $12 \mathrm{~h}$. This data suggests that the creatine kinases (CK, CK-MB) and the troponins (TnT, TnI) display differing release kinetic profiles in trimetazidine-treated CABG patients. Further trials that measure these cardiac markers across multiple time points after CABG and correlate these measurements with hard clinical outcomes are needed to better analyze trimetazidine's effects in CABG patients.

\section{Study Limitations}

Our systematic review has several limitations. First, study exclusion on the basis of language may have produced selection bias in the meta-analysis. For example, one large study including over 300 patients that was published in a Russian language journal by Lopatin and Dronova [37] was excluded on the basis of language and may have had a major impact on the current findings. Second, the number of included studies in each constituent meta-analysis was limited, and most of the included studies were relatively small in terms of sample size (i.e. with the exception of Sher-i-Murtaza et al. [19], all included studies had a sample size of under 100). Third, the included studies applied different protocols for preoperative trimetazidine therapy; the study of Vedrinne et al. [21] used a combination of oral and intravenous administration while the remaining studies solely used oral administration, and there were also differences in the duration of preoperative oral administration ranging from 1 day to 3 weeks prior to surgery (table 1). Fourth, the included studies cover a range of nearly two decades (1996-2014) in which the preoperative clinical management and surgical approaches to CABG patients have changed. Fifth, the studies used various types, manufacturers and generations of cardiac marker assays and employed various thresholds of significance. Sixth, there was a significantly high heterogeneity detected in the CK, CK$\mathrm{MB}$ and TnT meta-analyses. Seventh, the $2 \mathrm{TnI}$ studies $[23,24]$ failed to blind participants, which may have been 
Table 1. Characteristics of included trials

\begin{tabular}{|c|c|c|c|c|c|c|c|c|}
\hline \multirow[t]{2}{*}{ First author } & \multirow[t]{2}{*}{ Country } & \multicolumn{2}{|c|}{ Trimetazidine group } & \multicolumn{2}{|c|}{ Control group } & \multirow[t]{2}{*}{ Intervention } & \multirow[t]{2}{*}{ Follow-up } & \multirow{2}{*}{$\begin{array}{l}\text { Reported } \\
\text { outcomes }\end{array}$} \\
\hline & & $\begin{array}{l}\text { sample } \\
\text { size }\end{array}$ & age, years & $\begin{array}{l}\text { sample } \\
\text { size }\end{array}$ & age, years & & & \\
\hline $\begin{array}{l}\text { Sher-i-Murtaza } \\
\text { [19], } 2012\end{array}$ & Pakistan & 85 & $54.41 \pm 8.76$ & 85 & $54.8 \pm 10.79$ & $\begin{array}{l}20 \mathrm{mg} \text { orally preoperatively ( } 10 \mathrm{p} . \mathrm{m} \text {. at } \\
\text { night before day of surgery); } 20 \mathrm{mg} \text { orally } \\
\text { on the day of surgery }\end{array}$ & $36 \mathrm{~h}$ postoperatively & $\begin{array}{l}\mathrm{CK}-\mathrm{MB} \\
\mathrm{CPK}\end{array}$ \\
\hline $\begin{array}{l}\text { Tünerir [20], } \\
1999\end{array}$ & Turkey & 15 & $57.1 \pm 2.2$ & 15 & $58.4 \pm 1.2$ & $60 \mathrm{mg}$ q.d. for 3 weeks preoperatively & $48 \mathrm{~h}$ postoperatively & $\begin{array}{l}\text { CK-MB } \\
\text { TNT }\end{array}$ \\
\hline $\begin{array}{l}\text { Vedrinne [21], } \\
1996\end{array}$ & France & 20 & $61.0 \pm 2.0$ & 20 & $62.0 \pm 2.0$ & $\begin{array}{l}20 \mathrm{mg} \text { orally t.i.d. for } \geq 15 \text { days } \\
\text { preoperatively; } 40 \mathrm{mg} \text { as a bolus before skin } \\
\text { incision; } 2.5 \mathrm{mg} / \mathrm{h} \text { up to the sixth } \\
\text { postoperative hour }\end{array}$ & $24 \mathrm{~h}$ postoperatively & $\begin{array}{l}\mathrm{CK}-\mathrm{MB} \\
\mathrm{CK}\end{array}$ \\
\hline $\begin{array}{l}\text { Iskesen [22], } \\
2009\end{array}$ & Turkey & 15 & $57.5 \pm 2.6$ & 15 & $60.2 \pm 2.1$ & $\begin{array}{l}20 \mathrm{mg} \text { orally t.i.d. for } 2 \text { weeks } \\
\text { preoperatively; } 60 \mathrm{mg} \text { on the day of surgery }\end{array}$ & $48 \mathrm{~h}$ postoperatively & $\begin{array}{l}\text { TNT } \\
\text { CK }\end{array}$ \\
\hline $\begin{array}{l}\mathrm{Xu}[23] \\
2014\end{array}$ & China & 36 & $60.4 \pm 10.5$ & 36 & $62.3 \pm 11.3$ & $\begin{array}{l}20 \mathrm{mg} \text { orally t.i.d. for } 1 \text { week } \\
\text { preoperatively; } 60 \mathrm{mg} \text { on the day of surgery }\end{array}$ & $24 \mathrm{~h}$ postoperatively & TNI \\
\hline $\begin{array}{l}\text { Wang [24], } \\
2003\end{array}$ & China & 30 & $59.5 \pm 11.4$ & 30 & $62.1 \pm 9.6$ & $20 \mathrm{mg}$ orally t.i.d. for 3 weeks preoperatively & $24 \mathrm{~h}$ postoperatively & TNI \\
\hline
\end{tabular}

a source of bias in the TnI meta-analysis. Finally, we solely evaluated cardiac marker levels as opposed to more clinically relevant outcomes such as cardiovascular or allcause mortality. Therefore, if increased levels of a postCABG cardiac marker do not predict noncardiovascular mortality, our results would represent an overestimation of the association between preoperative trimetazidine therapy and cardiovascular mortality.

\section{Conclusions}

This systematic review and meta-analysis of 6 placebocontrolled RCTs investigated the effectiveness of preoperative trimetazidine therapy on myocardial preservation in CABG patients by assessing the postoperative levels of sev- eral blood-based biochemical markers of myocardial injury. Significantly lower postoperative levels of CK, CK-MB, $\mathrm{TnT}$ and $\mathrm{TnI}$ were found in the trimetazidine-treated CABG patients relative to control CABG patients. In sum, preoperative trimetazidine therapy appears to have a positive effect on myocardial preservation in CABG patients.

\section{Acknowledgments}

This study was supported by the Chongqing Science and Technology Commission (grant No. cstc2011ggcx0003).

\section{Conflicts of Interest}

None.

\section{References}

Varnauskas E: Twelve-year follow-up of survival in the randomized European Coronary Surgery Study. N Engl J Med 1988;319:332337.

2 Hoffman JW Jr, et al: Myocardial reperfusion injury: etiology, mechanisms, and therapies. J Extra Corpor Technol 2004;36:391-411.

-3 Weman SM, et al: Reperfusion injury associated with one-fourth of deaths after coronary artery bypass grafting. Ann Thorac Surg 2000; 70:807-812.
Moore GW, Hutchins GM: Coronary artery bypass grafts in 109 autopsied patients. Statistical analysis of graft and anastomosis patency and regional myocardial injury. JAMA 1981; 246:1785-1789.

5 Klatte K, et al: Increased mortality after coronary artery bypass graft surgery is associated with increased levels of postoperative creatine kinase-myocardial band isoenzyme release: results from the GUARDIAN trial. J Am Coll Cardiol 2001;38:1070-1077.
Lopaschuk GD, et al: Beneficial effects of trimetazidine in ex vivo working ischemic hearts are due to a stimulation of glucose oxidation secondary to inhibition of long-chain 3-ketoacyl coenzyme A thiolase. Circ Res 2003;93:e33-e37.

7 Danchin N, et al: Efficacy comparison of trimetazidine with therapeutic alternatives in stable angina pectoris: a network meta-analysis. Cardiology 2011;120:59-72. 
$>8 \mathrm{Hu}$ B, et al: Evaluation of trimetazidine in angina pectoris by echocardiography and radionuclide angiography: a meta-analysis of randomized, controlled trials. Clin Cardiol 2011; 34:395-400.

-9 Zhou X, Chen J: Is treatment with trimetazidine beneficial in patients with chronic heart failure? PLoS One 2014;9:e94660.

$>10$ Zhang L, et al: Additional use of trimetazidine in patients with chronic heart failure: a metaanalysis. J Am Coll Cardiol 2012;59:913-922.

-11 Liu Y, Li L, Su Q, Liu T, Tang Z: Trimetazidine pretreatment inhibits myocardial apoptosis and improves cardiac function. Cardiology 2015;130:130-136.

12 Kemp M, et al: Biochemical markers of myocardial injury. Br J Anaesth 2004;93:63-73.

$>13$ Liberati A, et al: The PRISMA statement for reporting systematic reviews and meta-analyses of studies that evaluate health care interventions: explanation and elaboration. J Clin Epidemiol 2009;62:e1-e34.

14 Higgins JPT, Green S: Cochrane handbook for systematic reviews of interventions version 5.1. 0 (updated March 2011). The Cochrane Collaboration, 2011. www.cochranehandbook.org.

15 Moher D, et al: Preferred reporting items for systematic reviews and meta-analyses: the PRISMA statement. Int J Surg 2010;8:336341.

16 Cohen J: Statistical Power Analysis for the Behavioral Sciences. New York, Routledge Academic, 2013.

$>17$ Higgins J, Thompson SG: Quantifying heterogeneity in a meta-analysis. Stat Med 2002; 21:1539-1558.

18 Higgins J, et al: Measuring inconsistency in meta-analyses. BMJ 2003;327:557-560.
19 Sher-i-Murtaza M, Zubair M, Jalal A: Is there any benefit of preoperative oral trimetazidine in coronary artery bypass graft? J Pak Med Assoc 2012;62:1271-1276.

20 Tünerir B, et al: Measurement of troponin T to detect cardioprotective effect of trimetazi dine during coronary artery bypass grafting. Ann Thorac Surg 1999;68:2173-2176.

21 Vedrinne J-M, et al: Myocardial protection during coronary artery bypass graft surgery: a randomized, double-blind, placebo-controlled study with trimetazidine. Anesth Analg 1996;82:712-718.

22 Iskesen I, et al: Trimetazidine may protect the myocardium during cardiac surgery. The Heart Surgery Forum 2009;12:175-179.

23 Xu P, Wen B, Jiao Z, Liu C, Zhao W: The cardioprotective effects of trimetazidine during off-pump coronary artery bypass grafting. Henan Med Res 2014;23:29-31.

24 Wang H, Hu D, Jia S: The cardioprotective efficacy of TMZ during coronary artery bypass graft surgery. Chin J Med Guide 2003;5:017.

25 Chrusciel P, Rysz J, Banach M: Defining the role of trimetazidine in the treatment of cardiovascular disorders: some insights on its role in heart failure and peripheral artery disease. Drugs 2014;74:971-980.

26 Marzilli M, Klein WW: Efficacy and tolerability of trimetazidine in stable angina: a metaanalysis of randomized, double-blind, controlled trials. Coron Artery Dis 2003;14:171179.

27 Ciapponi A, Pizarro R, Harrison J: Trimetazidine for stable angina. Cochrane Database Syst Rev 2005;4:CD003614.
28 Belsey J, et al: Relative efficacy of antianginal drugs used as add-on therapy in patients with stable angina: a systematic review and metaanalysis. Eur J Prev Cardiol 2014, Epub ahead of print.

29 Gao D, et al: Trimetazidine: a meta-analysis of randomised controlled trials in heart failure. Heart 2011;97:278-286.

30 Grande P, et al: Estimation of acute myocardial infarct size in man by serum CK-MB measurements. Circulation 1982;65:756764.

31 Babuin L, Jaffe AS: Troponin: the biomarker of choice for the detection of cardiac injury. Can Med Assoc J 2005;173:1191-1202.

-32 Adabag AS, et al: Prognostic significance of elevated cardiac troponin I after heart surgery. Ann Thorac Surg 2007;83:1744-1750.

33 Paparella D, et al: Myocardial injury after offpump coronary artery bypass grafting operation. Eur J Cardiothorac Surg 2007;32:481487.

34 Januzzi JL, et al: A comparison of cardiac troponin $\mathrm{T}$ and creatine kinase-MB for patient evaluation after cardiac surgery. J Am Coll Cardiol 2002;39:1518-1523.

-35 Males RG, Stephenson J, Harris P: Cardiac markers and point-of-care testing: a perfect fit. Crit Care Nurs Q 2001;24:54-61.

36 Petäjä L, et al: Biochemical injury markers and mortality after coronary artery bypass grafting: a systematic review. Ann Thorac Surg 2009;87:1981-1992.

37 Lopatin I, Dronova E: Clinical-pharmacoeconomic aspects of trimetazidine modified release use in patients with ischemic heart disease undergoing coronary artery bypass grafting (in Russian). Kardiologiia 2008;49: 15-21. 